\title{
Bronchotomy as a Safe and Feasible Alternative to Failed Bronchoscopic Removal of Foreign Body Inhalation in Pediatrics. A Seven-Year Review
}

\author{
Montaser Abd Elaziz'1,2, Mohammed Eltaweel1', Mohamed Elsayed², Mohammed El-Hag-Aly1 \\ ${ }^{1}$ Cardiothoracic Surgery Department, Faculty of Medicine, Menoufia University, Al Minufya, Egypt \\ ${ }^{2}$ Faculty of Medicine, Jazan University, Jazan, KSA \\ Email: montaserabdelaziz5@gmail.com,mnt_swy@yahoo.com, emansaleh364@gmail.com,eman_neuro_saleh@yahoo.com
}

How to cite this paper: Elaziz, M.A., Eltaweel, M., Elsayed, M. and El-Hag-Aly, M. (2021) Bronchotomy as a Safe and Feasible Alternative to Failed Bronchoscopic Removal of Foreign Body Inhalation in Pediatrics. A Seven-Year Review. Open Journal of Thoracic Surgery, 11, 47-57.

https://doi.org/10.4236/ojts.2021.112007

Received: May 10, 2021

Accepted: June 19, 2021

Published: June 22, 2021

Copyright $\odot 2021$ by author(s) and Scientific Research Publishing Inc. This work is licensed under the Creative Commons Attribution International License (CC BY 4.0).

http://creativecommons.org/licenses/by/4.0/

\begin{abstract}
Background: Aspiration of foreign body is popular in pediatric age group and is considered as an important cause of respiratory distress and chocking in these children. Management strategies are different according to the mode of presentation. This study aims to assess the efficacy of rigid bronchoscopy and the role of bronchotomy as a safe alternative for failed removal of inhaled foreign body. Methods: We enrolled 254 patients with foreign body aspiration (FBA) up to 14 years old in a retrospective observational study. Our patients were reviewed from the Cardiothoracic Surgery Department and cases referred from Otorhinolaryngology Department, Menoufia University Hospital between June 2010 and July 2017. Using jet ventilation technique, foreign body was removed by the rigid bronchoscopy with either extracting forceps or postural drainage. Surgical interference such as bronchotomy needed in distally impacted foreign bodies (FBs). Results: Our study included 254 patients prepared for bronchoscopic FBs extraction. Most of cases (68.8\%) presented early within first week (174 cases). No FBs detected in 44 cases by rigid bronchoscopy, although it was successful in 176 (69.5\%) cases with nonimpacted inhaled FBs. 14 cases only needed postural drainage due to inaccessible FBs, another 12 cases failed to extract FB with rigid bronchoscopy but they were managed with flexible bronchoscopy. Bronchotomy was needed for impacted FBs in eight cases (3\%). Conclusion: This study's findings support that rigid bronchoscopy is the gold standard in the diagnosis and removal of foreign body aspirations in pediatrics, but rigid bronchoscopy demonstrated less capability in the diagnosis and removal of small distally located foreign bodies. Bronchotomy is safe and effective alternative.
\end{abstract}




\section{Keywords}

Bronchoscopy, Bronchotomy, Foreign Body

\section{Introduction}

Inhalation of foreign body has high incidence during childhood. Flexible and rigid bronchoscopy is the mainstay of treatment for foreign body bronchus removal. However, when bronchoscopy fails, surgical removal of the foreign body bronchus via thoracotomy and bronchotomy is required in $2 \%$ of cases [1].

FBA patients may be symptomatic and present urgently for evaluation. Missed cases can be avoided by increasing our suspicion that our patient has FBA; this will aid in early diagnosis and management. Seventy percent of foreign body inhalation cases occur in children under the age of three [2].

Young children account for the majority of cases of FB inhalation into the respiratory system's airways. This is a life-threatening and potentially deadly disease that affects people all over the world. Modern bronchoscopic methods, pioneered by Gustav Killian, have resulted in lower mortality and morbidity rates after years of use [3].

If a clinical examination and chest x-ray fail to detect FBA, bronchoscopy may assist in the diagnosis of airway injury as a source of pulmonary problems induced by inhalational injury. Flexible fiberoptic bronchoscopy is useful for diagnosing FBA without inducing unwanted side effects [4].

Management differs depending on the mode of presentation. Children who have a partial airway obstruction and are at risk of deterioration despite maintaining their airway should be managed immediately in the operating room by removing the obstruction. For children with complete upper airway obstruction, basic life support must be administered. In stable conditions, we may need to re-evaluate our patients [5].

The aim of our study is to evaluate the diagnosis, and therapeutic bronchoscopic intervention, of foreign body aspiration, in children (up to 14 years old) and the role of bronchotomy as a safe alternative for failed removal of inhaled foreign body.

\section{Materials and Methods}

\subsection{Study Design}

Our retrospective observational study was carried out on the total of 254 children aged up to 14 years old; with suspected foreign body aspiration confirmed either by clinical presentation and/or radiological assessment and/or diagnostic bronchoscopy, although there were 44 children showed doubtful manifestations of FBA underwent diagnostic bronchoscopy which showed no FB detected in tracheo-bronchial tree.

Our patients were reviewed from Cardiothoracic Surgery Department and cases 
referred from Otorhinolaryngology Department after failure extraction of FB by rigid bronchoscopy in Menoufia University Hospital. The cases reviewed from a period between June 2010 and July 2017 were enrolled in the study. All patients were subjected to full history taking, general, chest and otorhinolaryngological examination plus plain chest X-ray.

A plain chest radiograph was routinely obtained in all suspected cases, but six patients underwent urgent bronchoscopic removal of the FB without undergoing pre-bronchoscopic radiography plain X-ray (2.37\%).

92 patients (36.4\%) had some significant clues in their histories and/or physical examinations, but they had negative radiographs, or the patients were too young to cooperate to produce an appropriate film. 24 computed tomography (CT) records of the chest were found. For children with suspicion but their history is a typical, clinical examination and chest-x ray did not aid to an accurate diagnosis, CT scan was performed.

The ethics committee in our institution approved the study. The patients' age and gender, symptoms and signs, duration of symptoms prior to bronchoscopy, radiological and bronchoscopic findings, including the type and location of the $\mathrm{FB}$, were all examined.

\subsection{Operative Technique}

\section{1) FB Extraction by rigid bronchoscopy}

All patients were subjected to inhalational anesthesia. The patients were preoxygenated with $100 \%$ Oxygen mask and then the anesthesia is induced by fentanyl $1: 2 \mathrm{mg} / \mathrm{kg}$ with propofol $2 \mathrm{mg} / \mathrm{kg}$. Before the introduction of the bronchoscope, $5 \%$ lidocine is sprayed into the trachea under laryngoscopic control. It is maintained by propofol infusion in a dose of $5 \mathrm{mg} / \mathrm{kg} \mathrm{ml}$ per hour with atracurium Besilate of $0.2 \mathrm{mg} / \mathrm{kg}$. The patients were ventilated either manually with very high flow oxygen or through traditional mechanical ventilation.

A rigid ventilating bronchoscopy with suitable size and light source was applied. The patient was put in the proper position when intubating the airway with a rigid bronchoscope. A tooth guard was placed to protect the upper incisors. The patient was in the supine position with atlanto-occipital extension. This position elevates the larynx and creates linear alignment of oral, pharyngeal and laryngeal axes. To properly inspect the vocal cords, the bronchoscopy was applied to elevate the epiglottis and under direct visualization it was advanced into the trachea. Or, if this was difficult, Machentouch laryngoscopy was done first then the appropriate size rigid bronchoscope was advanced into the trachea, ventilation was established and confirmed by the anesthesia team. The trachea was inspected completely till the primary carina. In order to properly visualize the left main bronchus, the bronchoscopy was set in the right angle of the mouth and the head of the patient was rotated to the right, to allign the long axis of the bronchoscope and left main bronchus. The patient's head and the bronchoscope were then rotated to the left to enter the right main bronchus. To avoid overrid- 
ing or displacement, the location of the foreign body was approached slowly and carefully. Finally, the forceps that were holding the FB were removed. Following the extraction of the detected FB, a second search was conducted for other FBs and residual FB fragments.

\section{2) Bronchotomy}

The choice of surgical removal of the foreign body through a thoracotomy was given to parents who consented to the procedure if FB extraction by rigid or flexible bronchoscopy failed. Fasting was observed, and the patient was given atropine $0.12 \mathrm{mg}$ intravenously (IV). The inhalational technique was used to induce anesthesia with oxygen, nitrous oxide, and $2 \%$ halothane. Succinyl choline $4 \mathrm{mg} \mathrm{IV}$ was used to relax the muscles, and the trachea was intubated with $3 \mathrm{~mm}$ uncuffed endotracheal tube (ETT).

The ETT was then purposefully manipulated into the main bronchus either right or left according to the location of $\mathrm{FB}$, if the FB was in the right side, the ETT was manipulated into the left main bronchus (unaffected side) until air entry was good on the unaffected side but absent on the affected one, and it was then secured. Flexible bronchoscopy was usually used to guide intubation. At all times, oxygen saturation was greater than 93 percent. The infant was carefully placed in the lateral decubitus position in preparation for a posterolateral thoracotomy on the affected side.

Thoracotomy and bronchotomy were performed, and the FB was delivered lengthwise through a small incision. Because of the uncuffed ETT used, there is minimal loss of ventilation during the bronchotomy, which is handled by the surgeon with intermittent manual occlusion of the defect until the defect is repaired.

We used interrupted absorbable sutures (Vicryl 3-0) for repair of bronchotomy. Bronchial patency should be checked, and the sutures were placed in a way that does not jeopardize the patency of the bronchus. Also anastomosis must be tension free. The anastomosis were protected by wrapping with a vascularized pedicle of autologous tissue (pleura, pericardial fat, or intercostal muscle). An intercostal flap should not cover 360 degrees of the circumference.

After that, the ETT was withdrawn into the trachea, and a leak test was performed to ensure that the bronchus was intact. The patient was extubated after reversing the neuromuscular block, ensuring strong respiratory efforts, intact airway reflexes, and normothermia. The patient was put in a humidified oxygen tent after surgery, and the chest tube was removed on day two. Patients were transferred to recovery unit until full recovery and improvement in cardiopulmonary parameters. Chest $\mathrm{x}$-ray was routinely done in our patients. We routinely perform a bronchoscopy just prior to the patient's discharge to assess the healing of the sutures.

\subsection{Statistical Analysis}

Data were coded, entered, and analyzed using using MedCalc ver. 15.8. (Med. Calc, Ostend, Belgium). Data were presented and statistically analysed according to type of each variable (parametric or non-parametric). P-values less than 
$0.05(5 \%)$ was considered to be statistically significant. Descriptive statistics were used in our study were frequency and percentage of non-numerical data plus mean, standard deviation $( \pm S D)$ and range for parametric numerical data, while median and inter-quartile range (IQR) for non-parametric numerical data.

\section{Results}

Our study was carried out on a total of 254 children aged up to 14 years old, According to age groups, 113 cases (44.48\%) were within three years ( 0 - 36 months) and 71 cases (27.95\%) were from 3 - 6 years ( 36 - 72 months). Majority of cases (61.42\%) were male (156 cases). $68.5 \%$ of cases were presented in the first week after onset of manifestation of FBA (Table 1).

The most presented clinical findings of it were choking (182 cases, $72 \%$ ) plus cough and diminished breath sounds (124 cases for each one, 49\%) as regards all clinical variables (Figure 1). Cyanosis is an alarming sign for FBA in cases (28 cases) presented with witnessed chocking that not needed to be confirmed with plain $\mathrm{x}$-ray so that they entered for emergent bronchoscopy.

Regarding the complications, we had 10 cases developed cardiac arrest, seven $(2.8 \%)$ of them were from early cases and three $(1.2 \%)$ were in delayed cases. Cardiac arrest was reversible in eight (3.2\%) cases, and was irreversible in 2 $(0.8 \%)$ cases. There was laryngeal edema in $28(11.1 \%)$ cases. Bronchotomy was an option in eight (3.2\%) cases (Table 2).

Among 254 cases included in our study, no FBs were detected in 44 cases either by radiographic investigations nor by bronchoscopy, there were only 210 cases with localized FBs. Rigid bronchoscopy was successful in extraction of FBs from 176 cases (69.5\%) with non-impacted inhaled FBs. 34 cases needed for another interference for removal of these FBs. These 34 cases showed detected FBs at segmental bronchi that were inaccessible by rigid bronchoscopy, so that postural drainage was done to these cases with lowering operating table head and suctioning

Table 1. Demographic \& Clinical data among 254 patients

\begin{tabular}{ccc}
\hline \multicolumn{2}{l}{ Variables } & $\begin{array}{c}\text { Median (inter-quartile range) } \\
\text { Frequency (\%) }\end{array}$ \\
\hline Gender & Male & $156(61.42 \%)$ \\
& Female & $98(38.58 \%)$ \\
\hline Age groups & $0-36$ months (0 - 3 years) & $113(44.48 \%)$ \\
& $36-72$ months (3 - 6 years) & $71(27.95 \%)$ \\
& $72-108$ months (6-9 years) & $46(18.11 \%)$ \\
\hline $\begin{array}{c}\text { Time of clinical } \\
\text { manifestations }\end{array}$ & $108-168$ months (9- 14 years) & $24(9.46 \%)$ \\
\hline & Early (First week) & $174(68.5 \%)$ \\
& Late (After first week) & $80(31.5 \%)$
\end{tabular}


Presentations of $\mathbf{2 5 4}$ patients in the study

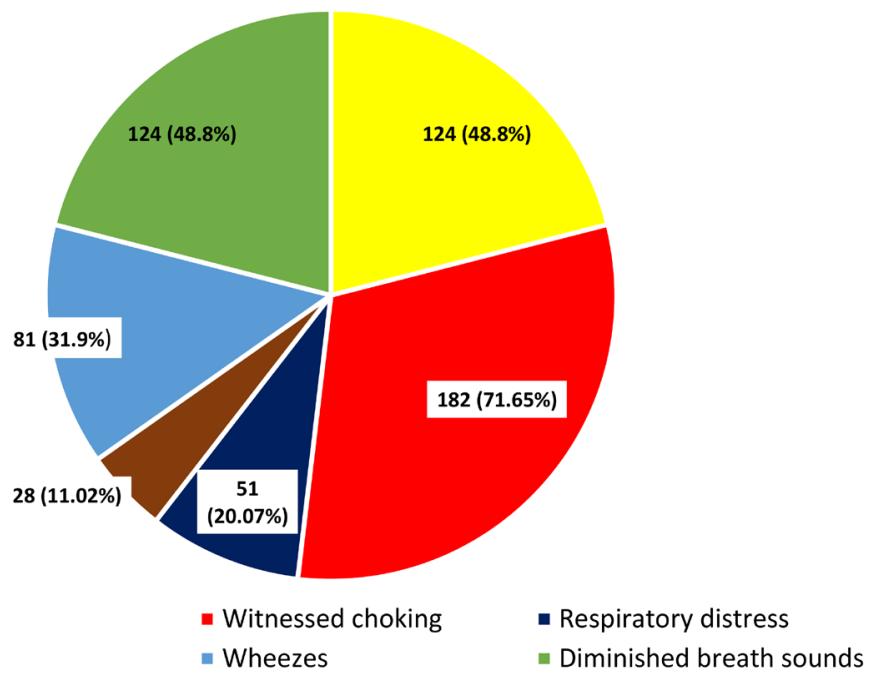

Figure 1. Clinical Manifestations of Patients in the study.

Table 2. Consequences after management.

\begin{tabular}{ccc}
\hline & \multicolumn{2}{c}{ (No. of patients = 254) } \\
\cline { 2 - 3 } Complication rate & $\begin{array}{c}\text { Early cases } \\
(138 \text { cases) No. (\%) }\end{array}$ & $\begin{array}{c}\text { Delayed cases (116 cases) } \\
\text { No. (\%) }\end{array}$ \\
\hline Cardiac Arrest & $7(2.8 \%)$ & $3(1.2 \%)$ \\
a) Reversible & $5(2 \%)$ & $3(1.2 \%)$ \\
b) Irreversible & $2(0.8 \%)$ & - \\
Laryngeal edema & $24(9.5 \%)$ & $4(1.6 \%)$ \\
Airway trauma & $1(0.4 \%)$ & $3(1.2 \%)$ \\
Bronchotomy & $6(2.4 \%)$ & $2(0.8 \%)$ \\
\hline
\end{tabular}

of these particles with closing the bronchoscopy as possible. This maneuver was successful in 14 cases, but failed to extract FBs in another 12 cases but they were managed with flexible bronchoscopy. Bronchotomy was needed at the same session for impacted distally located FBs in eight cases (3\%) that were presented mainly early (6 cases) or delayed ( 2 cases) (Figure 2 ).

Among 210 cases with localized FBs, 87 foreign bodies were located at left lung and 116 FBs were located at right lung plus another 7 of them were located bilaterally as regards side of FBs (Figure 3).

During bronchoscopy, 102 cases were found to have FB in the main bronchi either right or left, 74 in the lobar bronchus, and 34 cases in the segmental bronchi and rigid bronchoscopic extraction failed which needed further management (Figure 4).

Five cases who presented within the first week of clinical presentation had reversible cardiac arrested but two cases only had irreversible arrest. Only two cases of delayed presentation showed reversible arrest as shown in Table 2. 9.5\% 


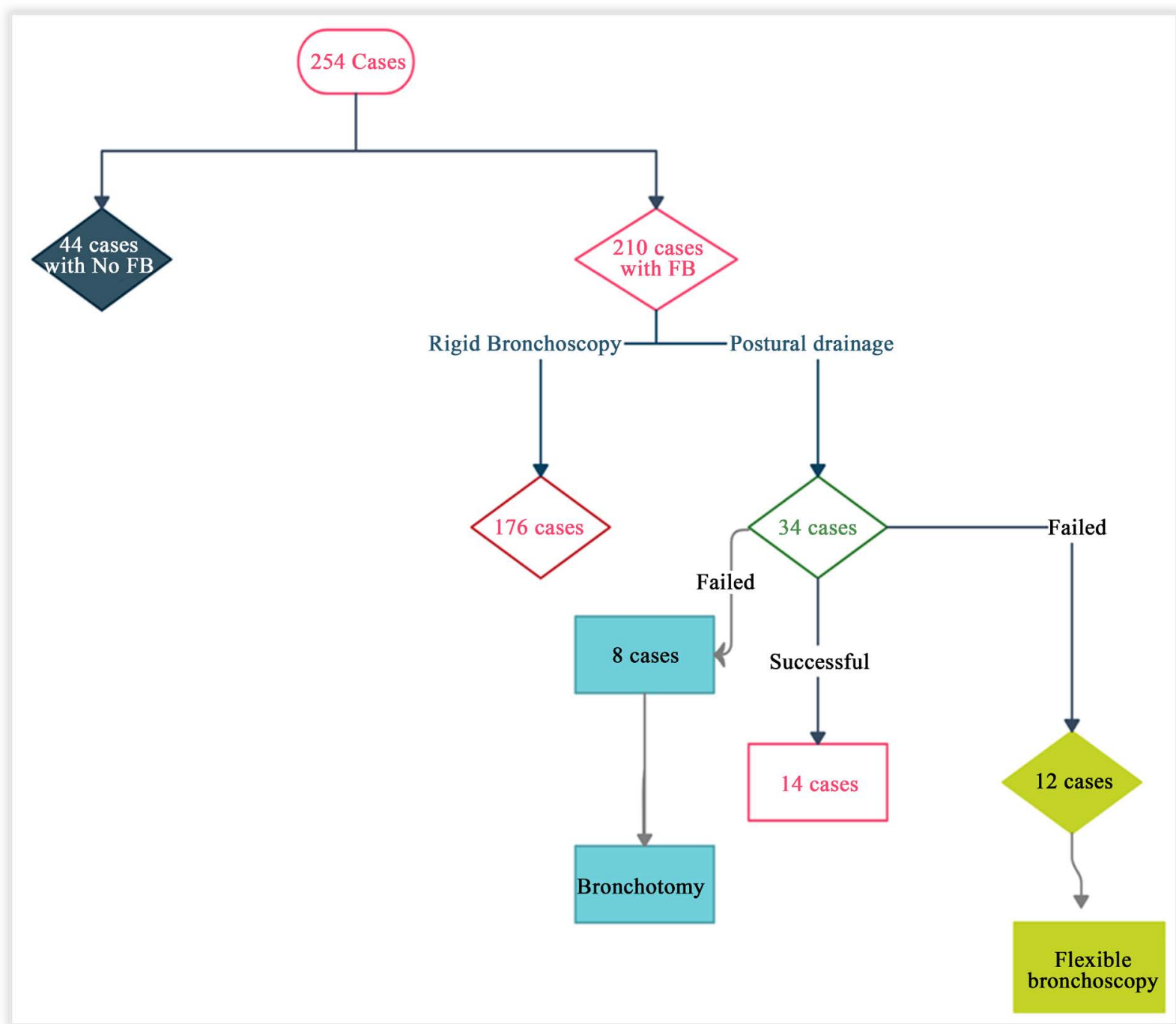

Figure 2. Flowchart of Patients's Managemement in the study.

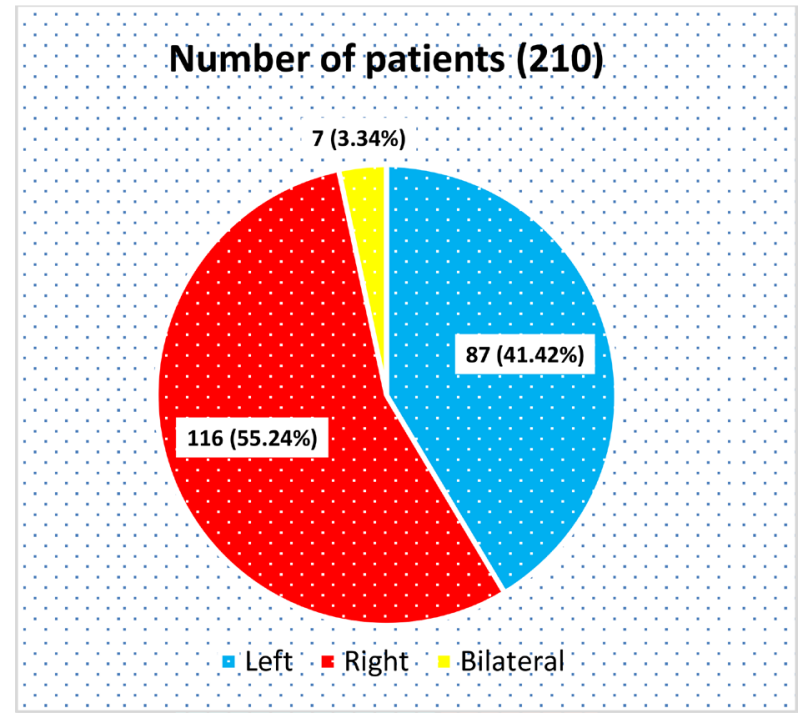

Figure 3. Sides of FB during bronchoscopy (210 cases). 


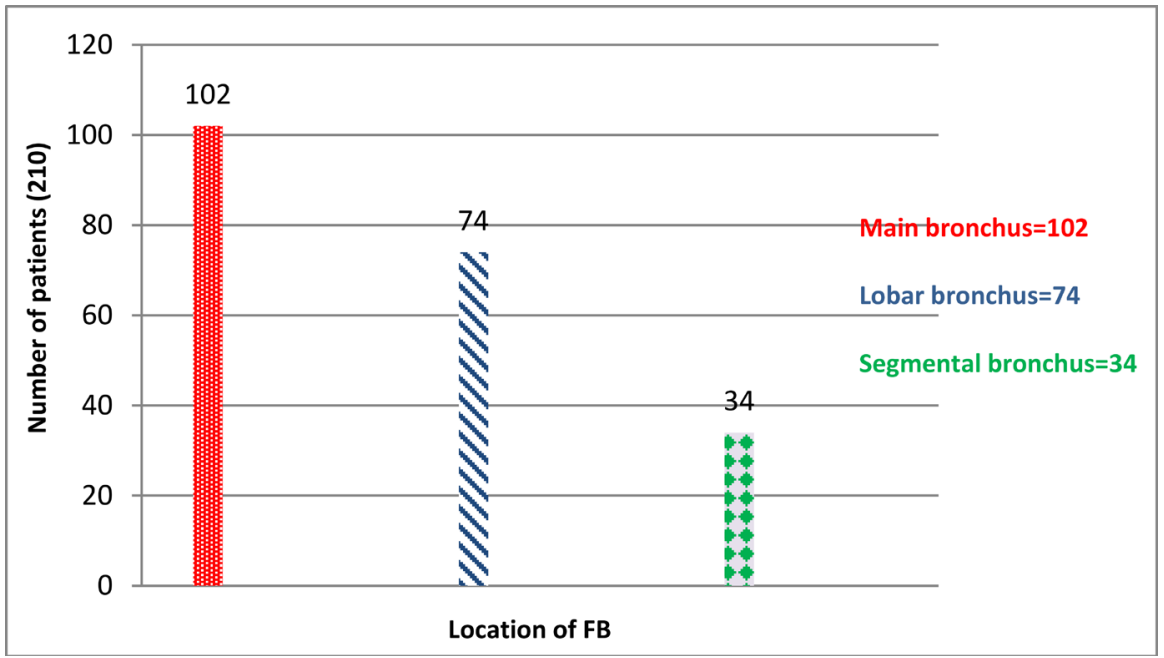

Figure 4. Locations of FB during bronchoscopy.

of cases presented postoperatively with biphasic stridor diagnosed as laryngeal edema ( 24 cases of early group and 4 cases of delayed group).

\section{Discussion}

Our study was done on 254 cases aged up to 14 years, while $44.7 \%$ of cases were up to 36 months. These results came in agreement with Sahin and his colleagues [2] who reported the fact that the highest prevalence of FB injuries was reported for children between zero and three years depends on that these group of children usually discover materials by mouth, and can not distinguish which of these materials is valid for eating or not.

Males were experienced to have FB injuries, therefore, boys seem to outnumber girls among infants with unintentional FB injury. Case series testify these airway FB injuries most commonly occur in young children [6].

FBs were found in 23 (33\%) of the 70 patients included in a study conducted by Haller et al. [7], (45 boys, 25 girls; median age: 21.5 months). They diagnosed FBA in 22 (96\%) and flexible bronchoscopy was used for extraction in seven of $23(30 \%)$, While in the remaining $16(70 \%)$ patients rigid bronchoscopy was used for the extraction of their FBs. They performed rigid bronchoscopy (as first intention) in $3 \%$ of patients. In patients confirmed to have FBs, the following signs were present: choking, apnea, cyanosis, diminished breath sounds, contralateral mediastinal shift, lung atelectasis, and air trapping. Conversely, when none of these symptoms or signs and no clear history of sudden choking were present (in 15/70 patients), no FB was found [7].

Another study showed that the most common presenting symptoms were cough (88\%), choking/gagging (67\%), and wheezing (57\%). Decreased breath sounds and wheezing on examination were independently associated with increased odds of a foreign body. Having a positive history, examination, and chest radiograph combined was $46 \%$ sensitive and $79 \%$ specific [8].

In a study conducted by Acharya, $32(80 \%)$ of 40 patients who underwent ri- 
gid bronchoscopy were found to have various foreign bodies in their airway, while 8 patients (20\%) had negative bronchoscopy. The only clinical symptom that was statistically significant ( $\mathrm{p}=0.043$ ) was a history of choking [9].

The study done by Kiyan on 192 patients who underwent rigid bronchoscopy, with the presence of wheeze as a symptom, had the highest sensitivity at $87.7 \%$, followed by unilateral decreased air entry on auscultation (i.e., 78.3\%) [10].

Hitter [11], demonstrated that a chest X-ray as an initial investigation had low specificity and sensitivity. Obstructive emphysema is the most specific radiographic sign in the absence of a radio-opaque foreign body. When radiographic signs are nonspecific, airway fluoroscopy can be used to complete the examination.

While rigid bronchoscopy is still the 'gold standard' for foreign body removal, it can fail due to impaction, peripheral position, or technical difficulty, necessitating a thoracotomy. In infants and teenagers, isolating the affected lung is technically challenging. Lung isolation is required to avoid blood contamination of the dependent lung and lack of ventilation during the bronchotomy. If the infected lung is not isolated, the foreign body can migrate into the opposite bronchus or into the trachea, resulting in complete ventilation failure [12].

A study of 400 cases found that flexible bronchoscopy had an 86 percent success rate [13]. If there is clinical suspicion of an airway FB in infants, some authors suggest going straight to bronchoscopy, even if the chest X-ray is negative [14].

In terms of bronchoscopic data, our findings were consistent with those of Belcher and colleagues [15], who claimed that rigid bronchoscopy is the gold standard for locating and extracting an aspirated FB.

In a study conducted by Goyal [16], 207 of 214 cases of foreign bodies confirmed by bronchoscopy were successfully extracted (96.7\%). Of these 214 cases, $105 \mathrm{FBs}$ (49\%) were found on the right side, $81 \mathrm{FBs}(38 \%)$ on the left side, and 28 FBs (13\%) in the trachea, These findings were consistent with that in our review, where we reported 176 cases (69.5\%) with successful rigid bronchoscopy, 34 cases failed extraction by rigid bronchoscopy, who were managed by flexible bronchoscopy (12 cases-), postural drainage (14 cases), and eight cases underwent thoracotomy and bronchotomy.

Also our findings agreed with those of Ambrose and colleagues [16], who found that while rigid bronchoscopy is the best procedure in the diagnosis of foreign body, flexible bronchoscopy had a much higher sensitivity for distal FB removal.

As regards the complication rate, our results came in agreement with a study of Ulla and colleagues [18]. They showed that complication rates during bronchoscopy was from $1 \%$ to $8 \%$, with a mortality rate of $<1 \%$. The most common complications included laryngeal edema, requiring intubation or tracheostomy, pneumothorax, pneumomediastinum, tracheal or bronchial laceration, failure to remove the foreign body, hypoxic brain injury, and cardiac arrest.

The study had some limitations as being retrospective in nature, and there was 
limited number of cases who underwent bronchotomy for furthur evaluation of the technique, but the limited percentage of bronchotomy cases in relation to the total number of patiens included in this study was accepted and consistent with other studies.

\section{Conclusion}

This study emphasizes that rigid bronchoscopy is considered the gold standard in the diagnosis and removal of FB aspirations in children (for large and small sized FBs), but rigid bronchoscopy demonstrated less capability in the diagnosis and removal of distally located FBs. Bronchotomy is safe and effective alternative required for difficult and failed bronchoscopic extraction especially in distally located FBs.

\section{Conflicts of Interest}

The authors declare no conflicts of interest regarding the publication of this paper.

\section{References}

[1] Causey, A.L., Talton, D.S., Miller, R.C. and Warren, E.T. (1997) Aspirated Safety Pin Requiring Thoracotomy: Report of a Case and Review. Pediatric Emergency Care, 13, 397-400. https://doi.org/10.1097/00006565-199712000-00011

[2] Sahin, A., Meteroglu, F., Eren, S. and Celik, Y. (2013) Inhalation of Foreign Bodies in Children: Experience of 22 Years. Journal of Trauma and Acute Care Surgery, 74, 658-663. https://doi.org/10.1097/TA.0b013e3182789520

[3] Becker, H.D. (2010) Bronchoscopy: The Past, the Present, and the Future. Clinics in Chest Medicine, 31, 1-18. https://doi.org/10.1016/j.ccm.2009.11.001

[4] Gad, S.S., Keshk, T.F., Khames, A.A., Elkashty, S.M. and Lasheen, A.F.A.E. (2018) Diagnostic Value of Bronchoscopy in Assessing the Severity of Inhalational Lung Injury. Menoufia Medical Journal, 31, 158.

[5] Orji, F.T. and Akpeh, J.O. (2010) Tracheobronchial Foreign Body Aspiration in Children: How Reliable Are Clinical and Radiological Signs in the Diagnosis? Clinical Otolaryngology, 35, 479-485. https://doi.org/10.1111/j.1749-4486.2010.02214.X

[6] Foltran, F., Ballali, S., Rodriguez, H., van As, A.B., Passali, D., Gulati, A. and Gregori, D. (2013) Inhaled Foreign Bodies in Children: A Global Perspective on Their Epidemiological, Clinical, and Preventive Aspects. Pediatric Pulmonology, 48, 344-351. https://doi.org/10.1002/ppul.22701

[7] Haller, L., Barazzone-Argiroffo, C., Vidal, I., Corbelli, R., Anooshiravani-Dumont, M. and Mornand, A. (2018) Safely Decreasing Rigid Bronchoscopies for ForeignBody Aspiration in Children: An Algorithm for the Emergency Department. European Journal of Pediatric Surgery, 28, 273-278. https://doi.org/10.1055/s-0037-1603523

[8] Sink, J.R., Kitsko, D.J., Georg, M.W., Winger, D.G. and Simons, J.P. (2016) Predictors of Foreign Body Aspiration in Children. Otolaryngology_Head and Neck Surgery, 155, 501-507. https://doi.org/10.1177/0194599816644410

[9] Acharya, K. (2016) Rigid Bronchoscopy in Airway Foreign Bodies: Value of the Clinical and Radiological Signs. International Archives of Otorhinolaryngology, 20, 
196-201. https://doi.org/10.1055/s-0036-1584293

[10] Kiyan, G., Gocmen, B., Tugtepe, H., Karakoc, F., Dagli, E. and Dagli, T.E. (2009) Foreign Body Aspiration in Children: The Value of Diagnostic Criteria. International Journal of Pediatric Otorhinolaryngology, 73, 963-967.

https://doi.org/10.1016/j.ijporl.2009.03.021

[11] Hitter, A., Hullo, E., Durand, C. and Righini, C.A. (2011) Diagnostic Value of Various Investigations in Children with Suspected Foreign Body Aspiration. European Annals of Otorhinolaryngology, Head and Neck Diseases, 128, 248-252. https://doi.org/10.1016/j.anorl.2010.12.011

[12] Ghosh, I. (1990) Delayed Removal of a Foreign Body in the Bronchus of a Child. Indian Journal of Anaesthesia, 38, 91-92.

[13] Silva, A.B., Muntz, H.R. and Clary, R. (1998) Utility of Conventional Radiography in the Diagnosis and Management of Pediatric Airway Foreign Bodies. Annals of Otology, Rhinology \& Laryngology, 107, 834-838.

https://doi.org/10.1177/000348949810701004

[14] Rafanan, A.L. and Mehta, A.C. (2001) Adult Airway Foreign Body Removal: What's New? Clinics in Chest Medicine, 22, 319-330.

https://doi.org/10.1016/S0272-5231(05)70046-0

[15] Belcher, R.H., Molter, D.W. and Goudy, S.L. (2018) An Evidence-Based Practical Approach to Pediatric Otolaryngology in the Developing World. Otolaryngologic Clinics of North America, 51, 607-617. https://doi.org/10.1016/j.otc.2018.01.007

[16] Goyal, R., Nayar, S., Gogia, P. and Garg, M. (2012) Extraction of Tracheobronchial Foreign Bodies in Children and Adults with Rigid and Flexible Bronchoscopy. Journal of Bronchology \& Interventional Pulmonology, 19, 35-43. https://doi.org/10.1097/LBR.0b013e318244e591

[17] Ambrose, S.E. and Raol, N.P. (2017) Pediatric Airway Foreign Body. Operative Techniques in Otolaryngology-Head and Neck Surgery, 28, 265-269. https://doi.org/10.1016/j.otot.2017.08.012

[18] Ullal, A., Mundra, R.K., Gupta, Y. and Mishra, S. (2019) Virtual Bronchoscopy: Highly Sensitive Time and Life Saving Investigation in the Diagnosis of Foreign Body Aspiration-Our Experience. Indian Journal of Otolaryngology and Head \& Neck Surgery, 71, 378-383. https://doi.org/10.1007/s12070-018-1319-2 